ZS Research Square

\title{
Development and Validation of Prognostic Nomograms for Patients with Parotid Gland Adenocarcinoma Not Otherwise Specified: A SEER Analysis From 2004 To 2016
}

\section{Zi-Meng Wang}

Tongji University Affilliated East Hospital: Shanghai East Hospital

Zuo-Lin Xiang ( $\nabla$ xiangzuolinmd@hotmail.com )

Tongji University Affilliated East Hospital: Shanghai East Hospital

\section{Research}

Keywords: Parotid gland, Adenocarcinoma not otherwise specified, SEER database, prognosis, nomogram

Posted Date: September 28th, 2021

DOI: https://doi.org/10.21203/rs.3.rs-915546/v1

License: () (1) This work is licensed under a Creative Commons Attribution 4.0 International License. Read Full License 


\section{Abstract \\ Background}

Parotid gland adenocarcinoma not otherwise specified (PANOS) is a rare malignancy, and the characteristics and prognosis of this disease remain unclear. This study aims to characterize PANOS and establish prognostic prediction models for patients with PANOS.

\section{Methods}

Cases from 2004-2016 were retrieved from the Surveillance, Epidemiology, and End Results Program database (SEER database). Univariate and multivariate Cox regression analyses, Gray's test and propensity score matching (PSM) were conducted to analyze demographics, treatments, and survival outcomes .

\section{Results}

The 446 patients ( 289 men) selected for analysis had a median age of 66 (19-95) years, and 307 patients were diagnosed with stage III/IV disease. The median survival of all patients was 66 months, with a 51.8\% 5year overall survival (OS) rate. Surgical treatment clearly improved survival time $(p<0.001)$. In the subgroup analysis, radiotherapy showed survival benefits in patients with advanced-stage disease (III/IV) $(p<0.001)$. Multivariate Cox regression analyses revealed that age, $\mathrm{T}$ stage, $\mathrm{N}$ stage, $\mathrm{M}$ stage and surgery were independent prognostic indicators for OS; $T$ stage, $\mathrm{N}$ stage, $\mathrm{M}$ stage and surgery were independent risk factors for cancer-specific survival(CSS).In addition, age was independently associated with noncancerrelated death. Two nomograms were established based on the results of the multivariate analysis, which was validated by the concordance index (C-index) ( 0.747 and 0.780 for OS and CSS, respectively) and the area under the time-dependent receiver operating characteristic(ROC) curve $(0.756,0.764$ and 0.819 regarding for nomograms predicting 3-, 5- and 10-year OS, respectively and $0.794,0.789$ and 0.806 for CSS, respectively).

\section{Conclusions}

Our study clearly presents the clinicopathological characteristics and survival analysis of patients with PANOS. In addition, our constructed nomogram prediction models may assist physicians in evaluating the individualized prognosis and deciding on treatment for patients.

\section{Background}

Parotid gland carcinoma(PGC) is a rare malignancy that accounts for $1 \%-3 \%$ of head and neck tumors ${ }^{[1]}$. Unlike the majority of head and neck malignancies being dominated by squamous cell carcinoma, PGCs are the most diverse, with 24 different histologic subtypes according to the 2017 World Health Organization (WHO) classification ${ }^{[2,3]}$. The most common pathological types of PGCs are mucinous epithelial carcinoma 
and adenoid cystic carcinoma, followed by adenocarcinoma not otherwise specified (ANOS) ${ }^{[4]}$. ANOS refers to carcinoma that has different levels of glandular differentiation in histology but cannot be attributed to a specific type. The biological behavior of ANOS is highly malignant, and it is prone to regional lymph node metastasis, and the local recurrence rate is also high ${ }^{[5,6]}$.

Parotid gland adenocarcinoma not otherwise specified (PANOS) is a sporadic malignancy arising from the parotid gland. Therefore, there are only a few published case reports or case series on the clinical characteristics and prognosis of PANOS. Li et al. ${ }^{[5]}$ reported that the mean overall survival (OS) of 11 patients with salivary adenocarcinoma not otherwise specified ( 7 were PANOS and 10 were high grade) was 15 months. Deng et al. ${ }^{[7]}$ reported that the 5- and 10-year OS rates of 28 patients with salivary adenocarcinoma not otherwise specified (14 were PANOS) were $62.2 \%$ and $36.0 \%$, respectively.

It is well known that the American Joint Committee on Cancer (AJCC) Staging is a commonly used prognostic tool for malignancies ${ }^{[8]}$. However, this staging system has been applied to entire populations, and is not necessarily highly predictive in individuals.Besides, it was based only on biological factors, excluding nonbiological factors such as age, sex, tumor differentiation and the treatments that patients have received. The nomogram includes a variety of cancer-related risk factors and can individually predict the survival rate of patients in a visual way ${ }^{[9,10]}$. In this study, we extracted cases from the SEER database from 2004 to 2016 to assess the clinicopathological characteristics of patients with PANOS and constructed two nomograms to directly predict the overall survival (OS) and cancer-specific survival (CSS) of these patients.

\section{Methods}

\section{Patients}

Data were extracted using SEER ${ }^{\star}$ Stat software (version 8.3.9) from the SEER Program (http://www.seer.cancer.gov) SEER*Stat Database: Incidence - SEER 18 Regs Custom Data (with additional treatment fields), Nov 2018 Sub (1975-2016 varying). Cases in which the parotid gland was the primary site were selected using the variable "primary site" (parotid gland $=079$ ). Cases in which adenocarcinoma not otherwise specified was the hisyologic type were identified by histology (International Classification of Diseases code: 8140/3). Cases were excluded if: the AJCC 6/7th TNM stage data were not complete; patients had a survival time of one month or less, were younger than 18 years old, were not first tumor and the grade, T stage, $\mathrm{N}$ stage, $\mathrm{M}$ stage, race, and laterality were unknown. Detailed information regarding age, gender, laterality, race, pathological grade, radiotherapy, chemotherapy, surgery, and the AJCC 6/7th TNM stage was extracted (Fig. 1).

\section{Statistical analysis}

The primary end point was OS and the secondary endpoint was CSS, which was defined as the period from diagnosis to death caused by PANOS or censoring. Univariate Cox regression analyses were conducted to filter out significant variables related to OS.Survival curves for each significant factor were depicted using the Kaplan-Meier method and compared using the log-rank test.Then, the significant factors were validated 
by competing risk analysis. The cumulative incidence function curves were plotted for each significant variable and performed Gray's test to recognize the differences in cancer-specific mortality among different subgroups. Multivariate Cox regression analyses were subsequently conducted .

One-to-one propensity score matching (PSM) analysis was used to adjust the interference of confounding factors on chemotherapy. Age, gender, race, laterality, marital status, pathological grade, T, N and M stages, stage, surgery and radiotherapy were covariates used for matching.

Nomograms were built on the results of multivariate analyses. Calibration curves were plotted to assess the agreement between the nomogram predicted survival rate and the observed survival rate. The receiver operating characteristic (ROC) curves were analyzed by measuring the performance of the constructed nomogram. The accuracy of the nomogram was analyzed using the $\mathrm{C}$-index and the area under the timedependent ROC curve (AUC).

All statistical analyses were performed using R. A probability value ( $p$ value) of $<0.05$ was considered statistically significant.

\section{Results}

\section{Patient Characteristics}

A total of 446 patients with PANOS were identified in the SEER database between 2004 and 2016 (Table 1). The median age of these patients was 66 (19-95) years old. There were 289 male patients and 157 female patients, with a male-to-female ratio of 1.84. Of 446 patients with TNM stage information, 139 and 307 patients had stage I/II and III/IV tumors, respectively. With respect to pathological differentiation, there was a propensity for poorly differentiated tumors, followed by moderately differentiated and undifferentiated tumors. There was no distant metastasis in $90.4 \%$ (403/446) of the patients, while $50.9 \%(227 / 446)$ of the patients had nodal involvement. Surgical resection was the primary treatment modality (409/446). Most patients (319/446) underwent radiotherapy, and 102/446 patients underwent chemotherapy.

\section{Survival Analysis}

The median OS among all patients was 66 months [95\% Cl (confidence interval): 56-97]. The 3-, 5-, and 10year OS rates were $63.1 \%, 51.8 \%$ and $36.7 \%$, respectively (Fig. 2A). The 3-, 5-, and 10-year CSS rates were $68.2 \%, 60.2 \%$ and $48.3 \%$, respectively (Fig. $2 B$ ). The OS shortened with increasing tumor stage $(p<0.001$,Fig. 4G). The 5-year OS of patients with stages I, II, III, and IV were $62.2 \%, 57.5 \%, 36.6 \%$ and $16.8 \%$, respectively. Undergoing surgical treatment clearly improved survival time $(p<0.001$, Fig. $4 \mathrm{H})$. The median OS with and without surgery was 74 (95\% Cl: 60-104) and 15 (95\% Cl: 9-27) months, respectively. Univariate and multivariate analyses showed that radiotherapy was not significantly correlated with OS $(p=0.278)$. However, in subgroup analysis, radiotherapy showed survival benefits in patients with advanced-stage disease (III/IV $)(p<0.001$,Fig. 3). 
According to univariate Cox regression analyses, as shown in Table 1 and Fig. 4 , age $(p<0.001)$, gender $(p=$ $0.002)$, pathologic grade $(p<0.001)$, T stage $(p<0.001)$, N stage $(p<0.001), \mathrm{M}$ stage $(p<0.001)$, stage $(p<$ $0.001)$, chemotherapy $(p<0.001)$, and surgery $(p<0.001)$ were significant factors related to OS.Furthermore, the analysis of the cancer-specific survival curve based on the competitive risk model is summarized in Fig. 5. The results were similar to those of the univariate analysis but excluded the variables age $(p=$ $0.080)$.Additionally, age $(p<0.001)$, T stage $(p<0.001)$ and stage $(p<0.001)$ were considered to be related to noncancer-related death, as shown in Table 3.

The significant factors were further investigated in multivariate analysis. The results showed that age $(p<$ $0.001)$, T stage $(p<0.001)$, N stage $(p<0.001)$, M stage $(p<0.001)$, and surgery $(p<0.001)$ were independent prognostic factors for OS, while sex $(p=0.630)$, pathologic grade $(p=0.174)$ and chemotherapy $(p=0.628)$ were not considered independent risk factors (Table 2). In addition, T stage $(p<0.001)$, N stage $(p<0.001)$, M stage $(p=0.002)$, and surgery $(p<0.001)$ were independent prognostic factors for CSS while age $(p<0.001)$ was an independent risk factor for noncancer-related death(Table 3).

\section{Propensity Score Matching}

According to the univariate analysis, chemotherapy did not improve the survival of patients. Instead, the survival time of patients was shortened after chemotherapy treatment $(p<0.001)$. To verify the effects of chemotherapy, we performed PSM based on variables described in the methods. Regular data showed that chemotherapy showed a trend toward male $(p<0.001)$, high grade $(p<0.001)$, large tumor size $(p<0.001)$, positive nodal metastasis $(p<0.001)$, distant metastasis $(p<0.001)$, advanced stage $(p<0.001)$, radiotherapy $(p=0.008)$ and no surgery $(p=0.001)$ (Table 4$)$. No significant differences in baseline features were observed in matched groups which included 82 patients. After PSM analysis, there was no significant difference in OS ( $p=0.6)$ between chemotherapy and no chemotherapy, and the median OS was 49 $(95 \% \mathrm{Cl}: 26-85)$ and 35 (95\% Cl:23-63) months, respectively (Fig. 6). There was no significant difference in $\operatorname{CSS}(p=0.9)$, either.

\section{Establishment And Validation Of The Nomogram}

The significant variables based on the final multivariate models were used to create the nomograms for OS and CSS. The prognostic nomogram for 3-, 5-, and 10-year OS was shown in Fig. 7A. The prognostic nomogram for 3-, 5-, and 10-year CSS was shown in Fig. 7B. By adding up the scores related to each variable and projecting total scores to the bottom scales, we were easily able to calculate the estimated 3-, 5-, and 10year OS and CSS probabilities. The C-index for the prediction of OS and CSS was 0.747 and 0.780 , indicating that the two nomograms are in good agreement with the actual observations for PANOS patients. As shown in Fig. 8, the calibration curves (bootstrapping, $n=1,000$ ) showed a good fit for the predicted survival probability with the actual survival probability at the 3-, 5-, and 10-year time points.

The time-dependent receiver operating characteristic (ROC) has been used widely to display sensitivity and specificity in predictive models. As shown in Fig. 9,the area under the curve (AUC) values of ROC were 0.756 , 
0.764 and 0.819 regarding for nomograms predicting 3-, 5- and 10-year OS, respectively. While the 3-, 5-, and 10 -year AUC values of the nomogram for CSS were $0.794,0.789$ and 0.806 , respectively.

\section{Discussion}

Most published reports have focused on the overall assessment of parotid gland carcinomas due to the paucity of patients ${ }^{[11-13]}$. Li et al. and Deng et al. summarized the clinicopathologic features of PANOS only in few cases ${ }^{[5,7]}$. In the present study, we analyzed data from a cohort of 446 patients with PANOS retrieved from the SEER database. Our findings may present a comprehensive perspective on the characteristics and prognosis of PANOS patients.

In our study, the median survival of all patients was 66 months. The 5-year and 10-year OS rates were $51.8 \%$ and $36.7 \%$, respectively, which were lower than those of mucinous epithelial carcinoma and adenoid cystic carcinoma ${ }^{[12-13]}$. In our study, some clinical features of PANOS were also in agreement with previously published findings, including a higher number of males, a propensity for high-grade tumors and more patients who presented with advanced stage III/IV disease ${ }^{[5,12-13]}$.

Surgery is still the mainstay of treatment for parotid gland carcinomas, and treatment paradigms involving surgery have considerable effects on patient survival ${ }^{[16-18]}$. In this PANOS study, surgery notably prolonged OS among patients with PANOS $(p<0.001)$. The median OS of surgery and nonsurgery PANOS patients was 74 (95\% Cl: 60-104) and 15 (95\% Cl: 9-27) months, respectively. Multivariate Cox analyses also showed that surgery was an independent favorable prognostic factor both for OS and CSS. Radiotherapy was recommended for PANOS patients with incomplete resection and for patients with advanced stage disease who were considered unfit for surgery ${ }^{[19]}$. Improved survival after postoperative radiotherapy was shown by Terharrd et al. in parotid carcinomas ${ }^{[20]}$. In our study, radiotherapy clearly prolonged os for advanced-stage PANOS. The median OS of radiotherapy-treated and nonradiotherapy stage III/IV PANOS patients was 48 (95\%Cl: 36-60) and 19 (95\% Cl: 16-32) months, respectively. However, we showed that chemotherapy had no effect on extending survival time in the matched groups after PSM analysis $(p=0.9)$, and this finding was also supported by some previous reports ${ }^{[21-22]}$.

Age is an independent factor of OS and noncancer-related death, but it has no effect on CSS .The results of competing risk analysis also demonstrates it.Younger PANOS patients may have a better prognosis, which was in accordance with the literature ${ }^{[6,12]}$.

Unsurprisingly, T, N, or M stages are independent prognostic factors both for OS and CSS .Higher T, N, or M stage were associated with worse survival.

Finally, two nomograms were constructed and validated to quantitatively predict the 3-, 5- and 10-year survival rates using the independent prognostic factors, which may assist physicians in evaluating patients' prognoses for PANOS.

This study has certain limitations. First, considerable information on pathological grade, $\mathrm{T}$ stage, $\mathrm{N}$ stage and marital status was lacking for patients $137,26,10$ and 27 , respectively, which may have led to the 
extraction of inaccurate OS and CSS for the patients. Second, the lack of data on various chemotherapy regimens and clinical symptoms, such as pain or tenderness, in the SEER database limited the ability to determine their influences on the prognosis of PANOS patients. Third, the patients were all from the USA; thus, the results might not be applicable to other populations.

\section{Conclusions}

In conclusion, we used a population-based method to present the clinicopathological characteristics and survival analysis of patients with PANOS based on data from the SEER database. We showed that age, $T$ stage, $\mathrm{N}$ stage, $\mathrm{M}$ stage and surgery were independent prognostic factors for OS; $\mathrm{T}$ stage, $\mathrm{N}$ stage, $\mathrm{M}$ stage and surgery were independent risk factors for CSS. In addition, age was independently associated with noncancer-related death.Furthermore, we developed two nomograms that effectively and visually predicted the 3-, 5- and 10-year OS and CSS in patients with PANOS, which will contribute to evaluating the individualized prognosis and guiding treatment of the patients.

\section{Abbreviations}

parotid gland adenocarcinoma not otherwise specified (PANOS)

Surveillance, Epidemiology, and End Results Program database (SEER database)

propensity score matching (PSM)

overall survival (OS)

cancer-specific survival(CSS)

concordance index (C-index)

receiver operating characteristic(ROC)

parotid gland carcinoma(PGC)

World Health Organization (WHO)

adenocarcinoma not otherwise specified (ANOS)

American Joint Committee on Cancer (AJCC)

the area under the time-dependent ROC curve (AUC)

\section{Declarations}

\section{Ethics statement}


Approval for use of all data was obtained by submitting a request to the SEER program. No approval by the institutional review board was sought since SEER is a public database.

\section{Competing Interests}

The authors have declared that no competing interest exists.

\section{Consent for publication}

Not applicable.

\section{Availability of data and materials}

The datasets supporting the conclusions of this article are available in the SEER database at https://seer.cancer.gov/seerstat/.

\section{Funding}

This work was supported by the National Natural Science Foundation of China (Grant No.81960525), Science and the Technology innovation project of Shanghai (Grant No.19DZ1930900), Pudong New Area Science and Technology Development Fund (Grant No.PKJ2018-Y02) and Natural Science Foundation of Jiangxi (Grant No. 20192BAB205071).

\section{Contributions}

Zuo-Lin Xiang conceived the study design.Zi-meng Wang did the data acquisition and analysed and drafted the manuscript.Revision of the manuscript was done by Zuo-Lin Xiang. All authors contributed toward data analysis,drafting and critically revising the paper and agree to be accountable for all aspects of the work.

\section{Acknowledgements}

The authors want to thank the SEER database (https://seer.cancer.gov/seerstat/.) for providing information on cancer statistics.

\section{References}

1. Kane WJ, McCaffrey TV, Olsen KD, et al. Primary parotid malignancies. A clinical and pathologic review. Arch Otolaryngol Head Neck Surg. 1991;117:307-15.

2. El-Naggar AK, Chan JK, Grandis JR, et al. World Health Organization classifcation of tumours. Chapter 7, tumours of salivary glands. Lyon: IARC press; 2017. pp. 160-202.

3. Xu F, Feng $X$, Zhao F, et al. Competing-risks nomograms for predicting cause-specific mortality in parotid-gland carcinoma: A population-based analysis. Cancer Med. 2021 Jun;10(11):3756-69.

4. Adelstein DJ, Koyfman SA, El-Naggar AK, et al. Biology and management of salivary gland cancers. Semin Radiat Oncol. 2012 Jul;22(3):245-53. 
5. Li J, Wang BY, Nelson M, et al. Salivary adenocarcinoma, not otherwise specified: a collection of orphans. Arch Pathol Lab Med. 2004;128:1385-94.

6. Tao L, Zhang D, Zhang M, et al. Clinical behaviours and prognoses of high- and low-risk parotid malignancies based on histology. Eur Arch Otorhinolaryngol. 2019 Feb;276(2):497-503.

7. Deng R, Tang E, Yang X, et al. Salivary adenocarcinoma, not otherwise specified: a clinicopathological study of 28 cases. Oral Surg Oral Med Oral Pathol Oral Radiol. 2012 May;113(5):655-60.

8. Zhang J, Pan Z, Zhao F, et al. Development and validation of a nomogram containing the prognostic determinants of chondrosarcoma based on the surveillance, epidemiology, and end results database. Int J Clin Oncol. 2019;24:1459-67.

9. Liang W, Zhang L, Jiang G, et al. Development and validation of a nomogram for predicting survival in patients with resected non-small-cell lung cancer. J Clin Oncol. 2015;33:861-9.

10. Balachandran VP, Gonen M, Smith JJ, et al. Nomograms inoncology: more than meets the eye. Lancet Oncol. 2015;16:e173-80.

11. Lima RA, Tavares MR, Dias FL, et al. Clinical prognostic factors in malignant parotid gland tumors. Otolaryngol Head Neck Surg. 2005 Nov;133(5):702-8.

12. Inaka Y, Kawata R, Haginomori SI, et al. Symptoms and signs of parotid tumors and their value for diagnosis and prognosis: a 20-year review at a single institution. Int J Clin Oncol. 2021 Jul;26(7):11708.

13. Molteni G, Molinari G, Ghirelli M, et al. Oncological outcomes of parotid gland malignancies: a retrospective analysis of 74 patients. J Stomatol Oral Maxillofac Surg. 2019 Sep;120(4):310-6.

14. Sun J, Sun Y, Yang F, et al.Nomogram to predict the prognosis of parotid gland mucoepidermoid carcinoma: a population-based study of 1306 cases. PeerJ 2019 Jul 1;7:e7237.

15. Mannelli G, Cecconi L, Fasolati M, et al. Parotid adenoid cystic carcinoma: Retrospective single institute analysis. Am J Otolaryngol. 2017 Jul-Aug;38(4):394-400.

16. Guzzo M, Locati LD, Prott FJ, et al. Major and minor salivary gland tumors. Crit Rev Oncol Hematol. 2010 May;74(2):134-48.

17. Shah SA, Riaz U, Zubair M, et al. Surgical presentation and outcome of parotid gland tumours. J Coll Physicians Surg Pak. 2013 Sep;23(9):625-8.

18. Lewis AG, Tong T, Maghami E. Diagnosis and Management of Malignant Salivary Gland Tumors of the Parotid Gland. Otolaryngol Clin North Am. 2016 Apr;49(2):343-80.

19. Bjørndal K, Krogdahl A, Therkildsen MH, et al. Salivary adenoid cystic carcinoma in Denmark 19902005: Outcome and independent prognostic factors including the benefit of radiotherapy. Results of the Danish Head and Neck Cancer Group (DAHANCA). Oral Oncol. 2015 Dec;51(12):1138-42.

20. Terhaard CH, Lubsen H, Rasch CR, et al; Dutch Head and Neck Oncology Cooperative Group. The role of radiotherapy in the treatment of malignant salivary gland tumors. Int J Radiat Oncol Biol Phys 2005 Jan 1;61(1):103-11.

21. Uijen MJM, Lassche G, van Engen-van Grunsven ACH, et al. Systemic therapy in the management of recurrent or metastatic salivary duct carcinoma: A systematic review. Cancer Treat Rev. 2020 
Sep;89:102069.

22. Wang X, Luo Y, Li M, et al. Management of salivary gland carcinomas - a review. Oncotarget 2017 Jan 17;8(3):3946-3956.

\section{Tables}

Due to technical limitations, table 1 is only available as a download in the Supplemental Files section. 
Table 2

Multivariate Cox regression analyses of OS and CSS.

\begin{tabular}{|c|c|c|c|c|c|c|}
\hline \multirow[t]{2}{*}{ Characteristics } & \multicolumn{3}{|c|}{ os } & \multicolumn{3}{|c|}{ css } \\
\hline & $p$ value & $\mathrm{HR}$ & $95 \% \mathrm{Cl}$ & $p$ value & $\mathrm{HR}$ & $95 \% \mathrm{Cl}$ \\
\hline Age, $\leq 66$ vs. $>66$ & $<0.001$ & 1.768 & $1.332-2.346$ & 0.060 & 1.768 & $1.332-2.346$ \\
\hline Gender,female vs. male & 0.630 & 0.917 & $0.670-1.256$ & 0.592 & 0.917 & $0.670-1.256$ \\
\hline Grade & 0.174 & 1.127 & $0.932-1.362$ & 0.250 & 1.127 & $0.932-1.362$ \\
\hline G1 & Ref & 1 & & Ref & 1 & \\
\hline G2 & 0.379 & 1.701 & $0.521-5.549$ & 1.478 & 0.677 & $0.346-6.311$ \\
\hline G3 & 0.093 & 2.716 & $0.847-8.712$ & 2.731 & 0.366 & $0.656-11.362$ \\
\hline G4 & 0.280 & 1.933 & $0.584-6.400$ & 1.732 & 0.577 & $0.400-7.490$ \\
\hline T stage & $<0.001$ & & & $<0.001$ & 1.467 & $1.280-1.680$ \\
\hline $\mathrm{T} 1$ & Ref & 1 & & Ref & 1 & \\
\hline $\mathrm{T} 2$ & 0.056 & 1.671 & $0.987-2.828$ & 0.057 & 1.931 & $0.981-3.804$ \\
\hline T3 & $<0.001$ & 2.578 & $1.502-4.425$ & 0.001 & 3.117 & $1.573-6.179$ \\
\hline $\mathrm{T} 4$ & $<0.001$ & 3.276 & $1.989-5.394$ & $<0.001$ & 4.282 & $2.264-8.100$ \\
\hline $\mathrm{N}$ stage & $<0.001$ & 1.457 & $1.243-1.707$ & $<0.001$ & 1.457 & $1.243-1.707$ \\
\hline NO & Ref & 1 & & Ref & 1 & \\
\hline N1 & 0.054 & 1.464 & $0.993-2.158$ & 0.067 & 1.546 & $0.970-2.461$ \\
\hline N2 & $<0.001$ & 1.861 & $1.310-2.645$ & $<0.001$ & 2.459 & $1.635-3.698$ \\
\hline N3 & 0.013 & 2.957 & $1.253-6.979$ & 0.005 & 3.961 & $1.520-10.318$ \\
\hline M stage M1vs. M0 & 0.002 & 2.059 & $1.286-3.295$ & 0.002 & 2.059 & $1.286-3.295$ \\
\hline Chemotherapy, yes vs. no & 0.628 & 1.090 & $0.758-1.567$ & 0.930 & 1.090 & $0.758-1.567$ \\
\hline Surgery,yes vs. no & $<0.001$ & 2.912 & $1.813-4.676$ & $<0.001$ & 2.912 & $1.813-4.676$ \\
\hline
\end{tabular}


Table 3

Multiple Cox regression of Noncancer-related death.

\begin{tabular}{|c|c|c|c|}
\hline & $p$ value & HR & $95 \% \mathrm{Cl}$ \\
\hline Age,$\leq 66$ vs. $>66$ & $<0.001$ & 1.768 & $1.332-2.346$ \\
\hline T stage & 0.503 & 0.176 & $0.086-0.357$ \\
\hline T1 & Ref & 1 & \\
\hline T2 & 0.125 & 3.422 & $0.170-16.490$ \\
\hline T3 & 0.202 & 2.705 & $0.587-12.457$ \\
\hline T4 & 0.162 & 3.187 & $0.627-16.197$ \\
\hline Stage & 0.704 & & \\
\hline I & Ref & 1 & \\
\hline II & 0.112 & 0.211 & $0.031-1.441$ \\
\hline III & 0.735 & 0.758 & $0.152-3.772$ \\
\hline IV & 0.403 & 0.481 & $0.086-1.675$ \\
\hline
\end{tabular}


Table 4

Baseline characteristics of patients divided by chemotherapy in the regular and matched groups.

\begin{tabular}{|c|c|c|c|c|c|c|}
\hline Characteristics & $\begin{array}{l}\text { No } \\
\text { chemotherapy } \\
N(\%) \\
N(\%)\end{array}$ & $\begin{array}{l}\text { Chemotherapy } \\
\mathrm{N}(\%)\end{array}$ & $p$ & $\begin{array}{l}\text { No } \\
\text { chemotherapy } \\
\mathrm{N}(\%)\end{array}$ & $\begin{array}{l}\text { Chemotherapy } \\
\mathrm{N}(\%)\end{array}$ & $p$ \\
\hline Total & $344(446)$ & $102(446)$ & & $82(164)$ & $82(164)$ & \\
\hline Age (year) & & & 0.231 & & & 0.525 \\
\hline$\leq 66$ & $177(51.5)$ & $60(58.8)$ & & $51(62.2)$ & $46(56.1)$ & \\
\hline$>66$ & $167(48.5)$ & $42(41.2)$ & & $31(37.8)$ & $36(43.9)$ & \\
\hline Gender & & & <. 001 & & & 0.853 \\
\hline Male & $208(60.5)$ & $81(79.4)$ & & $62(75.6)$ & $64(78.0)$ & \\
\hline Female & 136(39.5) & $21(20.6)$ & & $20(24.4)$ & 18(22.0) & \\
\hline Ethnicity & & & 0.164 & & & 0.870 \\
\hline White & $284(82.6)$ & $87(85.3)$ & & 73(89.0) & $71(86.6)$ & \\
\hline Black & $32(9.3)$ & 12(11.8) & & $7(8.5)$ & $9(11.0)$ & \\
\hline Other & $28(8.1)$ & $3(2.7)$ & & $2(2.4)$ & $2(2.4)$ & \\
\hline Laterality & & & 1 & & & 1.000 \\
\hline Left & $191(55.5)$ & $56(54.9)$ & & $44(53.7)$ & $43(52.4)$ & \\
\hline Right & $153(44.5)$ & $46(45.1)$ & & $38(46.3)$ & $39(47.6)$ & \\
\hline Marital status & & & 0.956 & & & 0.426 \\
\hline Married & $216(62.8)$ & $65(63.7)$ & & $52(63.4)$ & $46(56.1)$ & \\
\hline Divorced/other & 128(37.2) & $37(36.3)$ & & $30(36.6)$ & $36(43.9)$ & \\
\hline Grade & & & $\hat{0} .001$ & & & 0.567 \\
\hline G1 & $22(6.4)$ & $3(2.9)$ & & $2(2.4)$ & $3(3.7)$ & \\
\hline G2 & 108(31.4) & 14(13.7) & & 18(22.0) & $12(14.6)$ & \\
\hline G3 & $153(44.5)$ & $68(66.7)$ & & $46(56.1)$ & $53(64.6)$ & \\
\hline G4 & 61(17.7) & 17(16.7) & & 16(19.1) & 14(17.1) & \\
\hline T stage & & & $\dot{0} 001$ & & & 0.673 \\
\hline T1 & $92(26.7)$ & $8(7.8)$ & & $6(7.3)$ & $7(8.5)$ & \\
\hline
\end{tabular}




\begin{tabular}{|c|c|c|c|c|c|c|}
\hline Characteristics & $\begin{array}{l}\text { No } \\
\text { chemotherapy } \\
N(\%) \\
N(\%)\end{array}$ & $\begin{array}{l}\text { Chemotherapy } \\
\mathrm{N}(\%)\end{array}$ & $p$ & $\begin{array}{l}\text { No } \\
\text { chemotherapy } \\
\mathrm{N}(\%)\end{array}$ & $\begin{array}{l}\text { Chemotherapy } \\
\mathrm{N}(\%)\end{array}$ & $p$ \\
\hline $\mathrm{T} 2$ & $100(29.1)$ & 19(18.6) & & $20(24.4)$ & 16(19.5) & \\
\hline T3 & 61(17.7) & $27(26.5)$ & & $17(20.7)$ & $23(28.0)$ & \\
\hline T4 & $91(26.5)$ & $48(47.1)$ & & $39(47.6)$ & $36(43.9)$ & \\
\hline $\mathrm{N}$ stage & & & $\hat{0} .001$ & & & 0.364 \\
\hline NO & 198(57.6) & $21(20.6)$ & & $17(20.7)$ & 19(23.2) & \\
\hline N1 & $55(16.0)$ & $24(23.5)$ & & $21(25.6)$ & $20(24.4)$ & \\
\hline N2 & $86(25.0)$ & $56(54.9)$ & & $41(50.0)$ & $43(52.4)$ & \\
\hline N3 & $5(1.4)$ & $1(1.0)$ & & $3(3.7)$ & $0(0.0)$ & \\
\hline M stage & & & $\hat{0} .001$ & & & 0.803 \\
\hline MO & $328(95.3)$ & $75(73.5)$ & & $74(91.2)$ & $72(87.8)$ & \\
\hline M1 & $16(4.7)$ & $27(26.5)$ & & $8(9.8)$ & $10(12.2)$ & \\
\hline Stage & & & $\grave{0}_{0.001}$ & & & 0.914 \\
\hline I & $75(21.8)$ & $2(2.0)$ & & $2(2.4)$ & $1(1.2)$ & \\
\hline ॥ & $59(17.2)$ & $3(2.9)$ & & $3(3.7)$ & $3(3.7)$ & \\
\hline III & $59(17.2)$ & $10(9.8)$ & & $10(12.2)$ & $12(14.6)$ & \\
\hline IV & 151(43.8) & $87(85.3)$ & & 67(81.7) & $66(80.5)$ & \\
\hline Surgery & & & $\begin{array}{l}<.001 \\
0.0\end{array}$ & & & 0.811 \\
\hline Yes & $325(94.5)$ & $84(82.4)$ & & $73(89.0)$ & $71(86.6)$ & \\
\hline No & $19(5.5)$ & 18(17.6) & & $9(11.0)$ & $11(13.4)$ & \\
\hline Radiotherapy & & & 0.008 & & & 0.682 \\
\hline Yes & $235(68.3)$ & $84(82.4)$ & & $66(80.5)$ & $69(84.1)$ & \\
\hline No & 109(31.7) & 18(17.6) & & 16(19.5) & 13(15.9) & \\
\hline
\end{tabular}

\section{Figures}




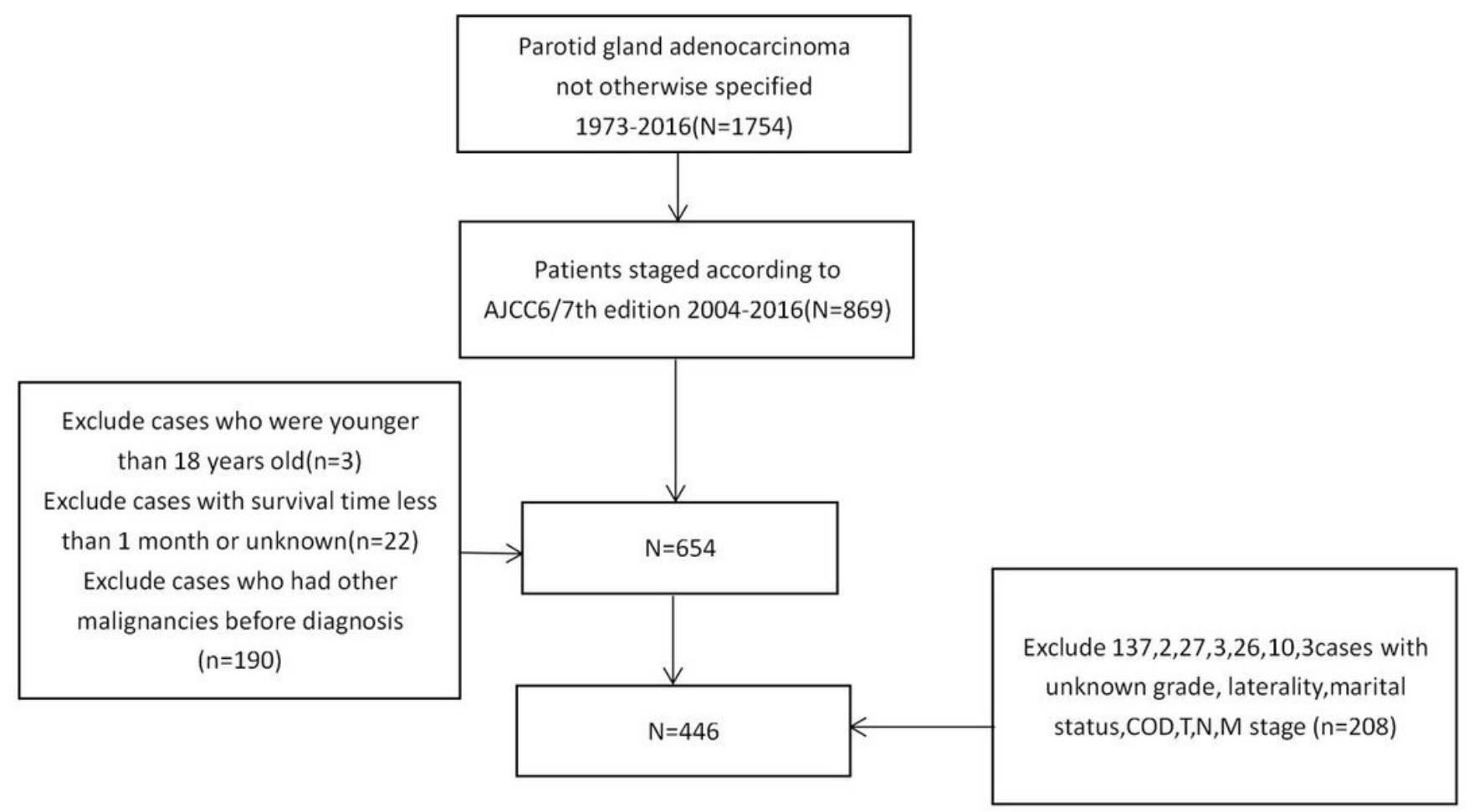

\section{Figure 1}

The flow diagram of the selection process for the study cohort.
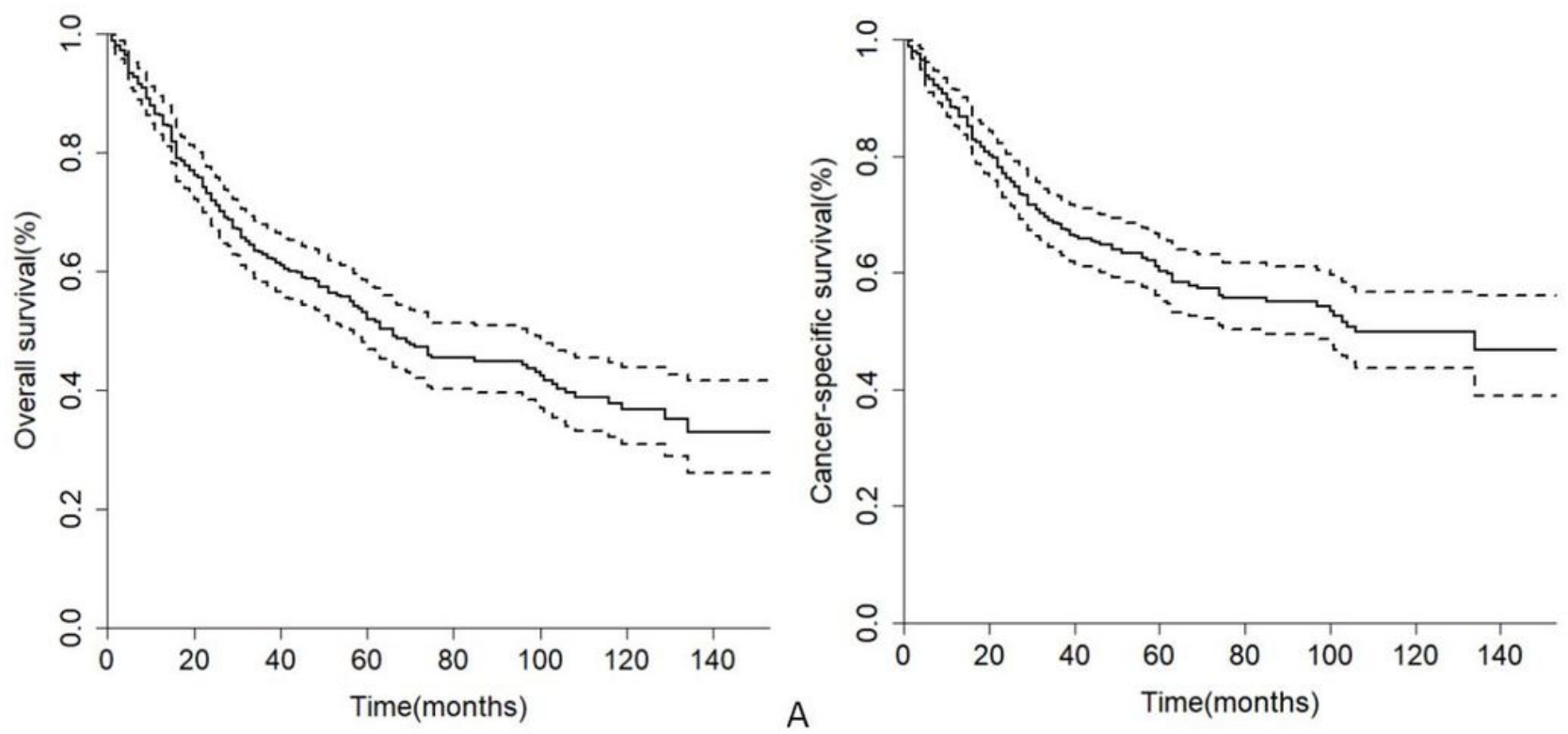

\section{Figure 2}


Kaplan-Meier curves of the 446 patients in the cohort .Overall survival(A);Cancer-specific survival(B).
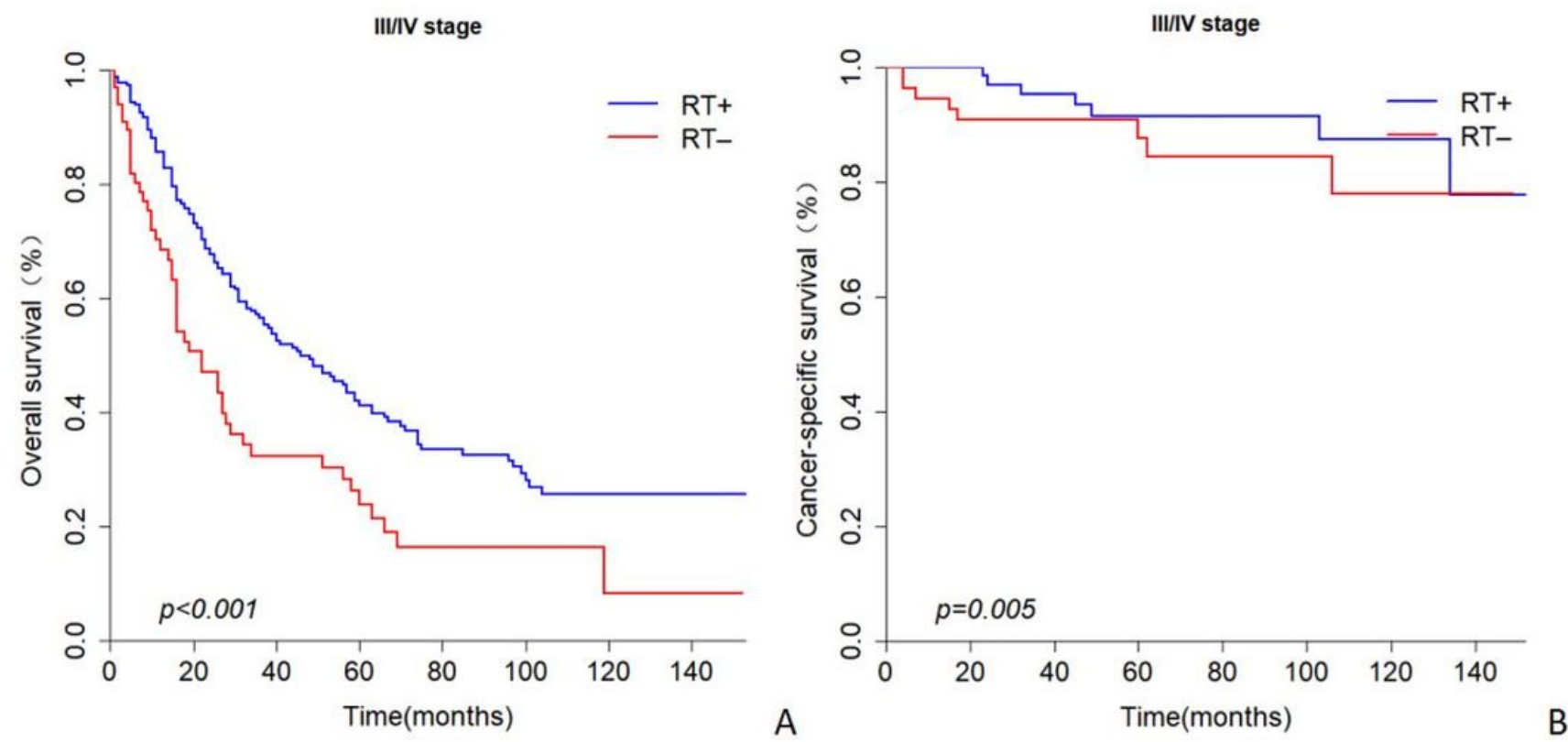

Figure 3

Kaplan-Meier curves of OS(A) and CSS(B) based on radiotherapy. 

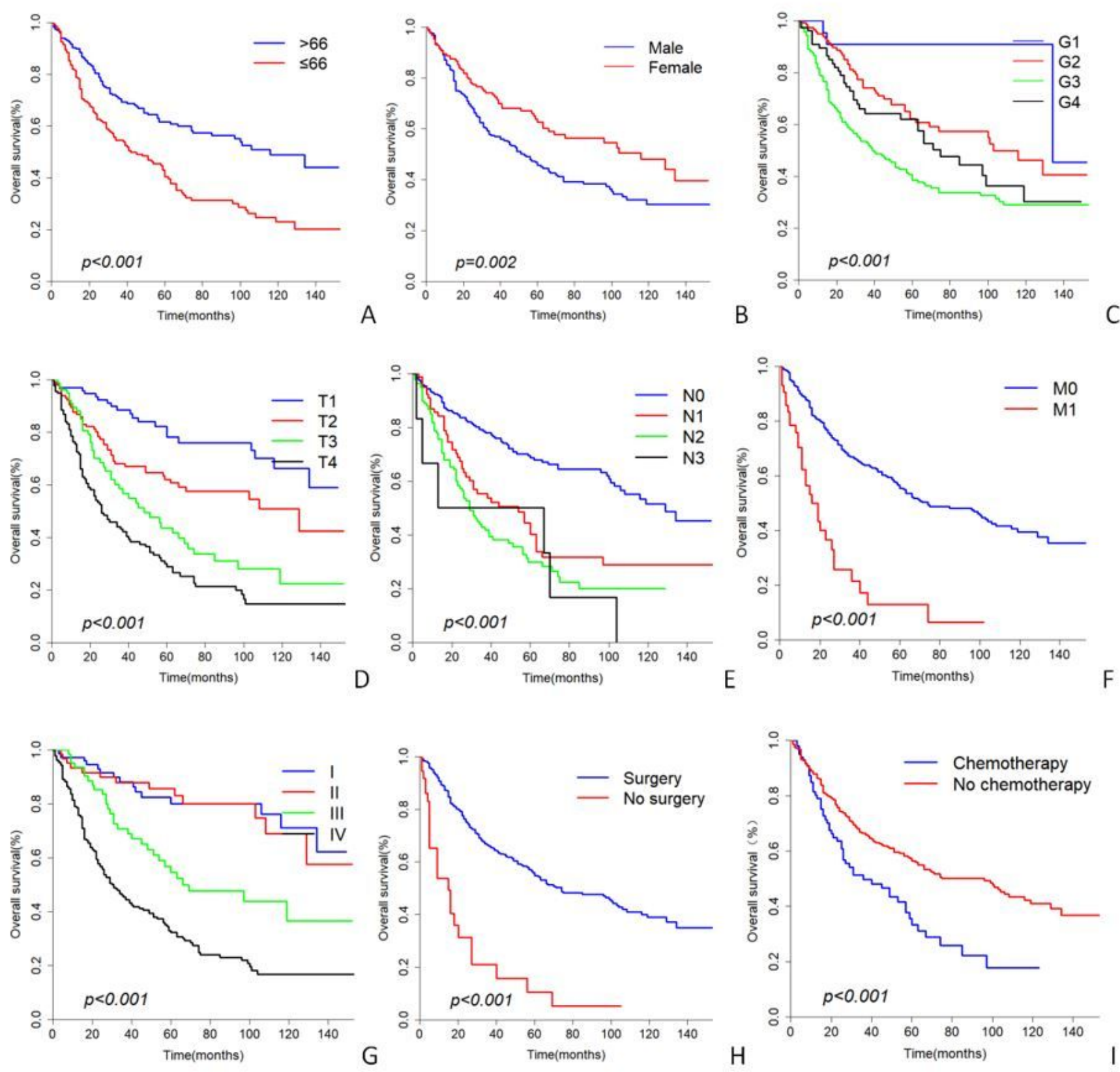

Figure 4

Overall survival curves according to (A) Age, (B) Gender, (C囚Grade, (D) T stage, (E) N stage, (F) M stage, (G) Stage, (H)Surgery, (I) Chemotherapy. 

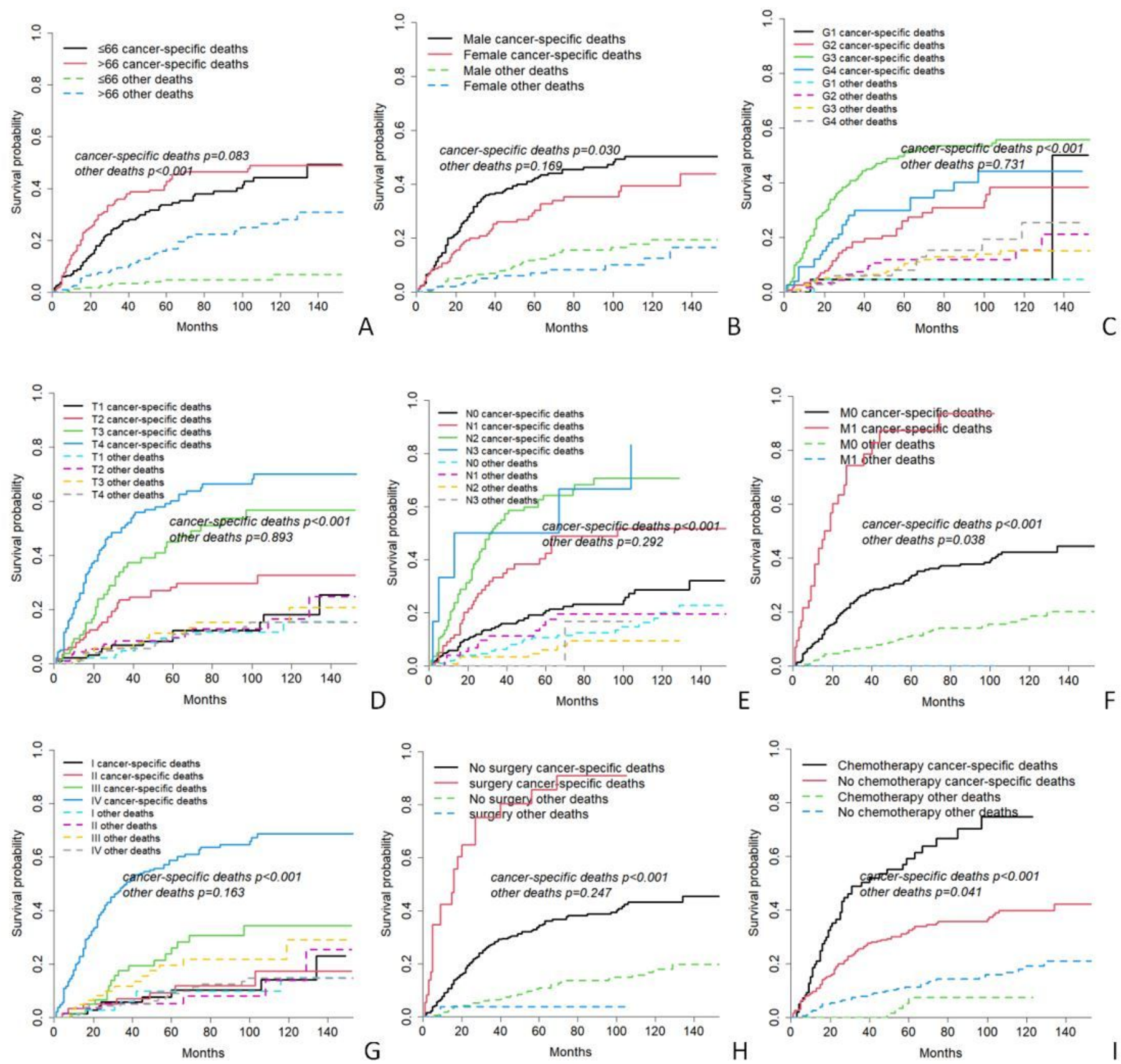

\section{Figure 5}

Competing risk analyses according to (A) Age, (B) Gender, (C凶Grade, (D) T stage, (E) N stage, (F) M stage, (G) Stage, (H)Surgery, (I) Chemotherapy. 

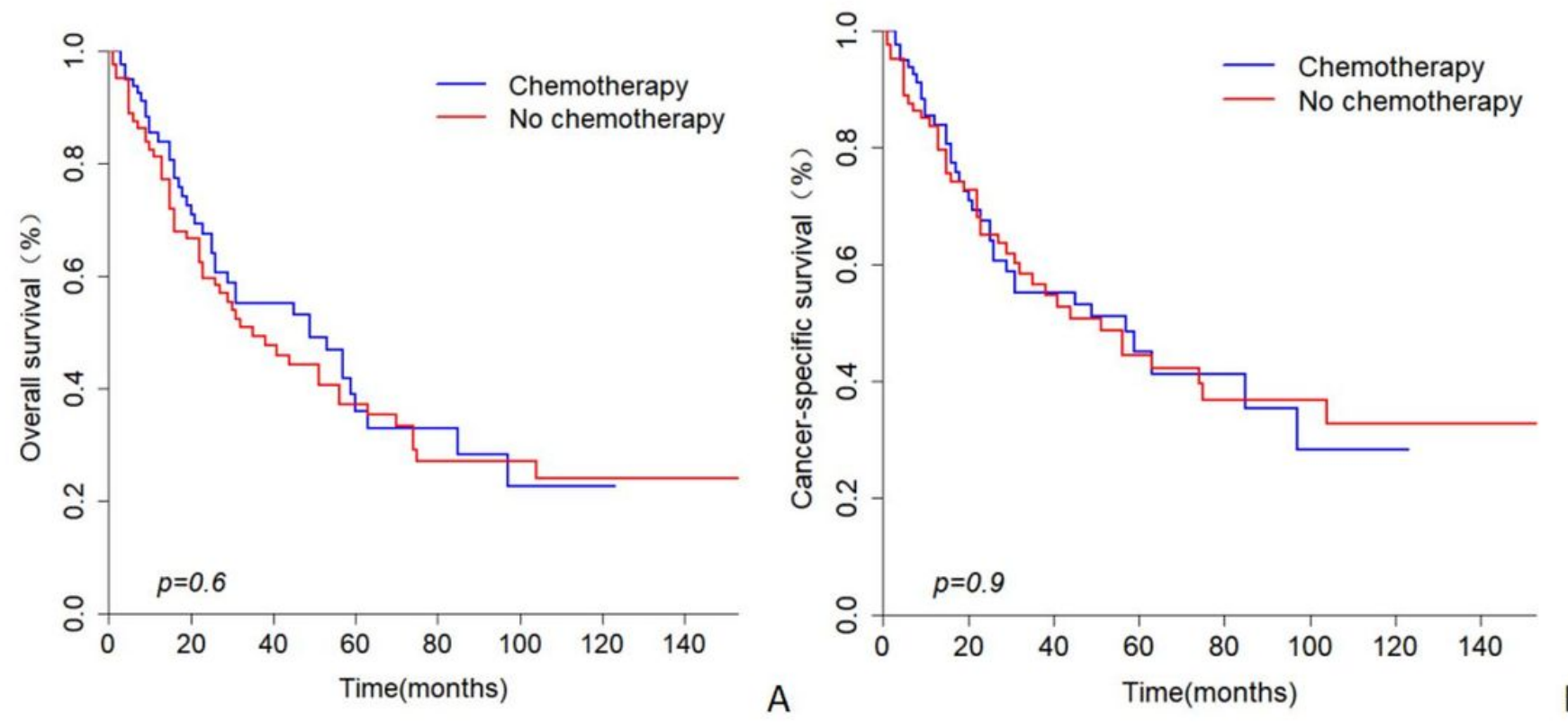

\section{Figure 6}

Kaplan-Meier curves of OS(A) and CSS(B) based on chemotherapy after PSM analysis. 


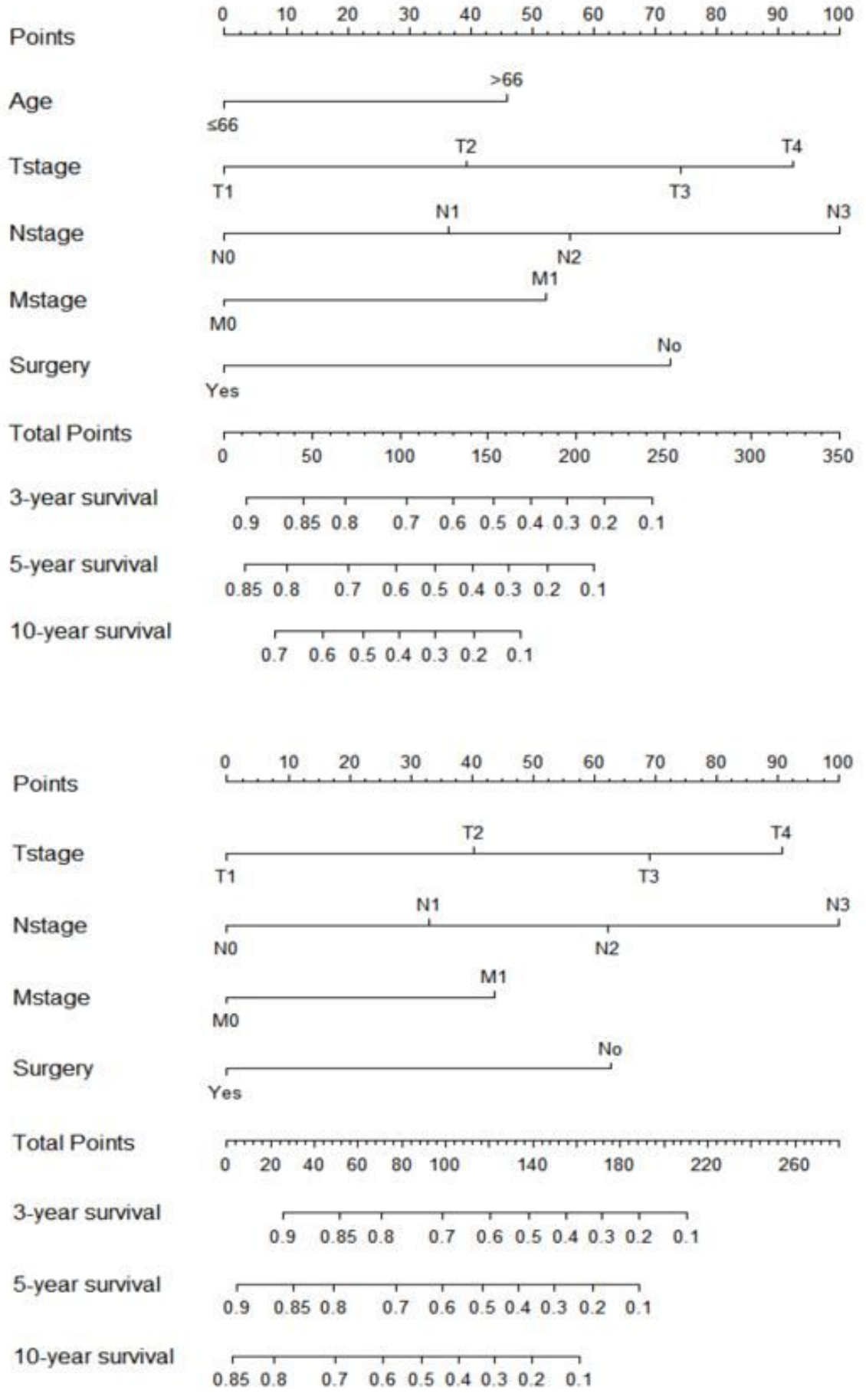

\section{Figure 7}

Nomograms for prediction of 3-, 5- and 10-year (A) OS rates ;(B) CSS rates. 

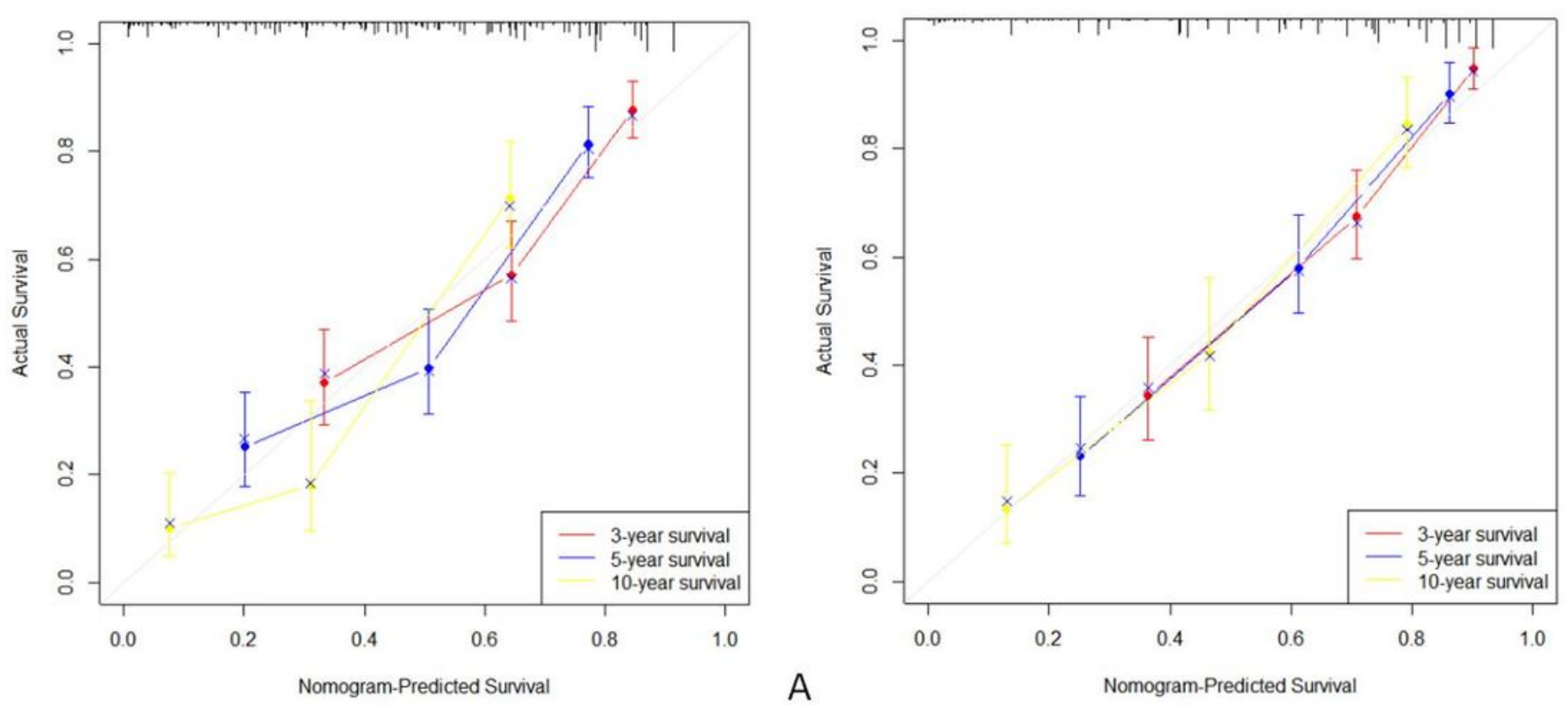

B

Figure 8

Calibration curves of the nomogram predicting 3-,5- and 10-year (A) OS rates ;(B) CSS rates.
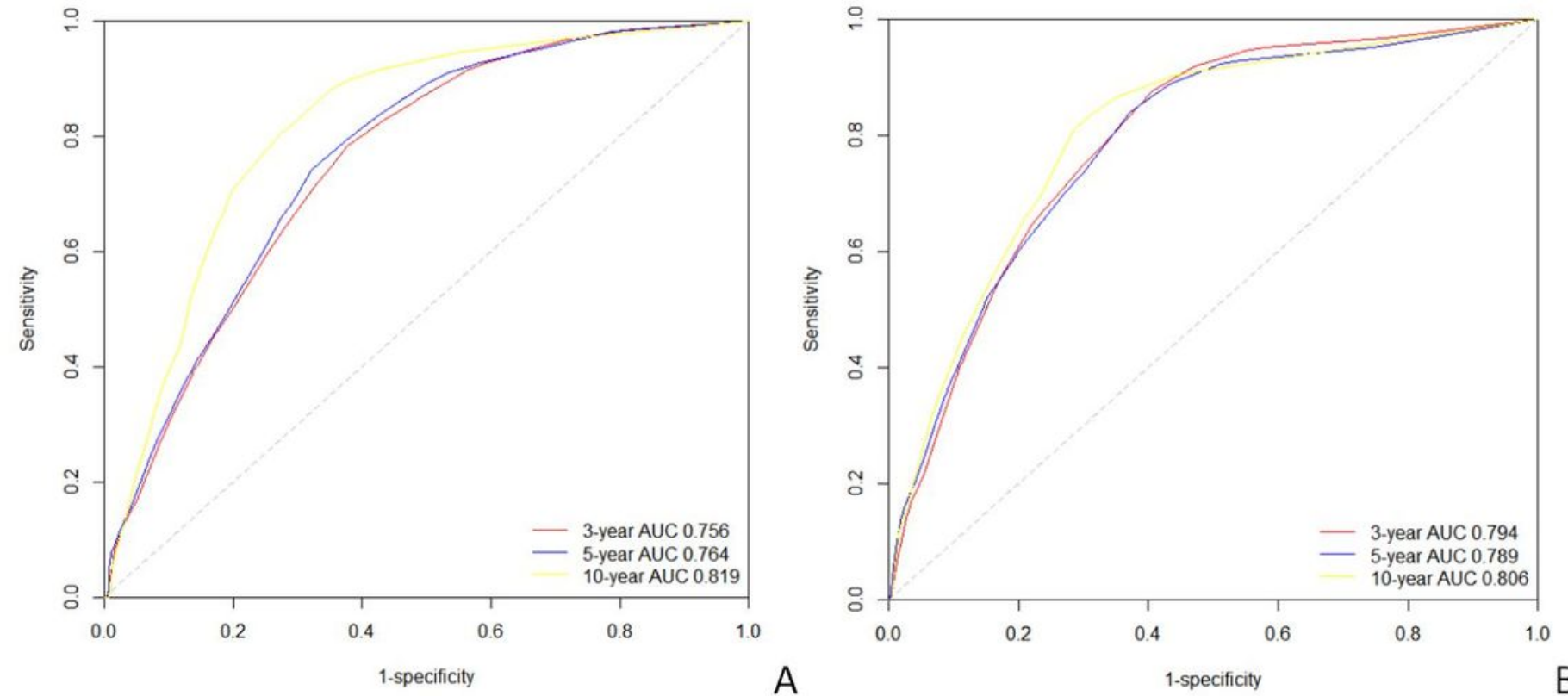

\section{Figure 9}

The time-dependent ROC curves of the nomograms predicting 3-,5- and 10-year (A) OS rates ; (B) CSS rates.

\section{Supplementary Files}


This is a list of supplementary files associated with this preprint. Click to download.

- Table1.jpg 